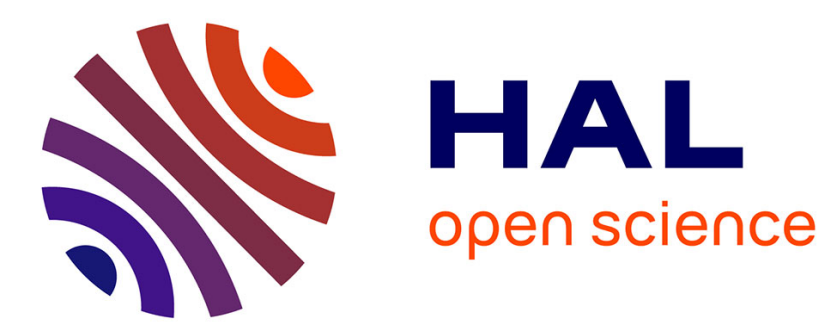

\title{
Positive control of Lotka-Volterra systems
}

Frédéric Grognard, Jean-Luc Gouzé

\section{To cite this version:}

Frédéric Grognard, Jean-Luc Gouzé. Positive control of Lotka-Volterra systems. 16th IFAC World

Congress, Jul 2005, Prague, Czech Republic. 10.3182/20050703-6-CZ-1902.00724 hal-01091732

\section{HAL Id: hal-01091732 \\ https://hal.inria.fr/hal-01091732}

Submitted on 9 Dec 2014

HAL is a multi-disciplinary open access archive for the deposit and dissemination of scientific research documents, whether they are published or not. The documents may come from teaching and research institutions in France or abroad, or from public or private research centers.
L'archive ouverte pluridisciplinaire HAL, est destinée au dépôt et à la diffusion de documents scientifiques de niveau recherche, publiés ou non, émanant des établissements d'enseignement et de recherche français ou étrangers, des laboratoires publics ou privés. 


\title{
Positive control of Lotka-Volterra systems
}

\author{
Grognard, J.-L. Gouzé \\ INRIA Sophia-Antipolis, BP 93 \\ 06902 Sophia-Antipolis CEDEX, FRANCE \\ frederic.grognardeinria.fr
}

July 2005

\begin{abstract}
We consider the $n$-dimensional Lotka-Volterra system of differential equation representing the predator-prey interaction between $n$ species. In this paper, we will try to control the system by the mean of a sign-definite control law that is based on a classical Lyapunov function for Lotka-Volterra-systems.Copyright (C)2005 IFAC Keywords: Predator-Prey, Lotka-Volterra, Positive control, Lyapunov design.
\end{abstract}

\section{Introduction}

In order to describe the interaction between $n$ species that are being the predator or the prey of each other, the Lotka-Volterra model

$$
\dot{x}=\operatorname{diag}(x)(A x+a)
$$

is often used (where $x \in \mathbb{R}_{+}^{n}, a \in \mathbb{R}^{n}, A \in \mathbb{R}^{n \times n}$, and $\operatorname{diag}(x)$ is a matrix containing the elements of the vector $x$ on its diagonal). This system is obviously positive. The vector $a$ describes the individual growth or death-rate of each species (when $a_{i}>0$, the species is growing in the absence of any other interaction; when $a_{i}<0$, it is dying). The $\operatorname{diag}(x) A x$ term represents the quadratic interaction between the species. In the original Volterra paper [8], it was supposed that $A$ was a skew symmetric matrix. Indeed, this assumption stems from the observation that, if species $i$ is the predator of species $j$, the species $i$ is benefiting from the predation in the quadratic form $+a_{i j} x_{i} x_{j}$, while the prey is being consumed at the rate $-a_{i j} x_{i} x_{j}$, both terms of the interaction having the same magnitude in order to satisfy the mass-balance of the interaction (when the $x_{i}(t)$ state represents the mass of the ith species at time $t$ ).

Most of the work that has been devoted to Lotka-Volterra systems mainly concentrates on the analysis of the behavior of the solutions. In this paper, we will introduce some control to influence the behavior of the system. We will concentrate on a control action that can only act on the growth rates of the species, so that the model becomes

$$
\dot{x}=\operatorname{diag}(x)(A x+a+b u)
$$


where $b \in \mathbb{R}^{n}$ and $u \in \mathbb{R}^{+}$. The $u$ term can be seen as the rate of application of a pesticide on a field, the rate of release of a predator, or a single factor that influences the growth-rate of all the species, like lightning or temperature. We will suppose that this control action is always non-negative: this is quite natural for the rate of application of pesticide, for the rate of release of a predator (if we assume that they cannot be caught afterwards), for the light and temperature, if the only actuator that we have are a lighting or a heating system. We will not assume that all the elements of $b$ have the same sign, so that the same control action can benefit a species $i$ (where $b_{i}>0$ ) and harm a species $j$ (where $b_{j}<0$ ). Such a control objective was already pursued in $[2,7]$ through optimal control theory, but those were limited to two-dimensional systems; a preliminary study of accessibility for n-dimensional Lotka-Volterra systems was however given in [1] while a first controller for those $\mathrm{n}$-dimensional systems was given in [4].

The behavior of the uncontrolled system (1) is well documented (see [8, 3]), and citing the work of Volterra: If there exists an equilibrium, and if the initial state is different from it, there will be fluctuations in the numbers of at least some of the species, and these fluctuations will not dampen. If those fluctuations occur for the pest species, it will most often result in peaks of the pest population that are higher than the pest population at the equilibrium. The control action will then be used to steer the solutions of the Lotka-Volterra system to the positive equilibrium of the uncontrolled system (that we denote $e \in \mathbb{R}_{+}^{n}$ ), so that those peaks are not present anymore and the pest population is monitored.

The existence of such an equilibrium implies that system (2) can be rewritten as

$$
\dot{x}=\operatorname{diag}(x)(A(x-e)+b u)
$$

where $a=-A e$. We will build a controller $u(x)$ that will ensure global asymptotic stability of $e$ inside the positive orthant with $u$ non-negative.

The paper is structured as follows: in Section 2, we will study the controllability of system (3) and show that, in many cases, the system is uncontrollable with a positive feedback. In Section 3, we will develop a controller (for the cases where noncontrollability has not been shown), and we will illustrate its action in simulations in Section 4. Finally we will give some conclusions.

\section{Controllability of Lotka-Volterra systems with non- negative controls}

In this paper, we will concentrate on the Lotka-Volterra predator-prey system

$$
\dot{x}=\operatorname{diag}(x)(A(x-e)+b u)
$$

where $x, e \in \mathbb{R}_{+}^{n}, b \in \mathbb{R}^{n}, A \in \mathbb{R}^{n \times n}$ is skew-symmetric and $u \in \mathbb{R}^{+}$.

In this section, we will outline cases where system (4) is not controllable so that, in those cases, we will not try and build a controller for the stabilization of the equilibrium. Indeed, we can show the following proposition 
Proposition 1 If $\operatorname{det}(A)=0$, then system (4) is not controllable with $u \geq 0$. Moreover, for any neighborhood $N$ of e, there exists $x_{0} \in N$ such that $x(t)$ (with initial condition in $x_{0}$ ) cannot be steered to e with non-negative control.

Proof: If $\operatorname{det}(A)=0$, one of the eigenvalues of $A$ is zero, to which corresponds a left eigenvector $v$ such that $v^{T} A=0$. We will now show that $e$ is not reachable for some initial conditions in the positive orthant. Indeed, let us consider the time-derivative of the function

$$
U(x)=v^{T} \ln (x)
$$

along the solutions of (4) (where $\left.\ln (x)=\left[\ln \left(x_{1}\right), \cdots, \ln \left(x_{n}\right)\right]^{T}\right)$. This results in

$$
\dot{U}=v^{T}(A(x-e)+b u)=v^{T} b u
$$

If we suppose, without loss of generality, that $v^{T} b \geq 0, U$ can only increase along the solutions of (4) when the control action is constrained to being non-negative. As a consequence, if $U(x(0))>U(e)$, no control $u(t) \geq 0$ can steer $x(t)$ to $e$. As the gradient of $U$ is non-zero at $e$, it is obvious that we can find $x(0)$ as close as we want to $e$ and such that $U(x(0))>U(e)$. For good measure, we will now build such a $x(0)$ and show that it can be made as close as we want to $e$, so that the system is not even locally controllable in $e$.

Let us fix $x_{j}(0)=e_{j}+\epsilon$ for all $j$ such that $v_{j} \geq 0$ and $x_{j}(0)=e_{j}-\epsilon$ for all $j$ such that $v_{j}<0$. With that choice, it is clear that

$$
U(x(0))>U(e)
$$

for any given $0<\epsilon<\min _{j \in\{1, \cdots, n\}}\left(e_{j}\right)$. Therefore, we have that $U(x(t)) \geq$ $U(x(0))>U(e)$ for all $t>0$, so that $x(t)$ cannot converge towards $e$. It is also clear that we can take $\epsilon$ as small as we want and still show that $e$ cannot be reached from $x(0)$, so that there exists no neighborhood $N$ of the equilibrium such that all of its elements can be steered to $e$.

It should be noted that Proposition 1 results in the non-controllability of the desired equilibrium when $n$ is odd. Indeed, in this case, the skew-symmetric matrix $A$ always has an eigenvalue in 0 .

Moreover, when $\operatorname{det}(A)=0$, the existence of a positive equilibrium for (4) results in the existence of a continuum of equilibria

$$
x^{*}=e+\alpha v
$$

with $v$ an eigenvector corresponding to the eigenvalue in 0 and $\alpha \in \mathbb{R}$. The choice of $e$ in the transformation of system (1) into (2) is therefore not unique.

There is a chance that some kind of controller can be build to steer $x(t)$ from $x_{0}$ to $e$ when $U\left(x_{0}\right)<U(e)$ and $v^{T} b>0$, but this would not result in stability of the equilibrium, and any kind of uncertainty could drive $x(t)$ in the region where $U(x(t))>U(e)$, resulting in the impossibility of bringing $x(t)$ back to $e$.

In the remainder of this text, we will consider that $A$ is invertible. 


\section{Control design}

In this section, we still consider system (4), but we now suppose that $A$ is invertible. This directly implies that $n$ is even. In this case, it is well known that system (4), with $u=0$, has a first integral:

$$
V(x)=\mathbf{1}^{T} x-e^{T} \ln (x)
$$

where $\mathbf{1}=\left[\begin{array}{lll}1 & \cdots & \cdots\end{array}\right]^{T}$. Indeed, the time-derivative of this functions is:

$$
\begin{aligned}
\dot{V} & =\mathbf{1}^{T} \operatorname{diag}(x)(A(x-e)+b u)-e^{T}(A(x-e)+b u) \\
& =x^{T}(A(x-e)+b u)-e^{T}(A(x-e)+b u) \\
& =(x-e)^{T} A(x-e)+(x-e)^{T} b u \\
& =(x-e)^{T} b u
\end{aligned}
$$

which is zero when $u=0$.

Using $V$ as a Control Lyapunov Function, we will render its derivative negative semi-definite. This is simply achieved with a feedback in the form

$$
u=\sigma\left(-b^{T}(x-e)\right)
$$

where $\sigma(s)=0$ when $s \leq 0$ and $s \sigma(s)>0$ when $s>0$. This feedback results in the following evolution of the first integral:

$$
\dot{V}=(x-e)^{T} b \sigma\left(-b^{T}(x-e)\right)
$$

which is negative if $b^{T}(x-e)<0$ and 0 otherwise. We can then show the following result

Proposition 2 The solutions of system (4) (with A invertible) with control (5) converge to the hyperplane $b^{T}(x-e)=0$. Moreover, in the case where $n=2$ and in the case where $n=4$ and $b=\alpha \mathbf{1}$ (for $\alpha \in \mathbb{R}$ ), the equilibrium $e$ is globally asymptotically stable

Proof: We have seen that the control (5) makes the time-derivative of the Lyapunov function $V$ negative semi-definite. We will then conclude the analysis through LaSalle's invariance principle that states that the solutions of system (4) (with controller (5)) starting in a positively invariant compact set $\Omega$ will converge towards the largest invariant set of the region where $\dot{V}=0$ inside $\Omega$. In this case, we can take the region surrounded by the level-set $V(x)=V_{\Omega}$ as compact set $\Omega$ (for some $V_{\Omega}>V(e)$ ). This region is positively invariant, so that LaSalle's principle can be applied and the solutions converge to the largest invariant set of the region where $\dot{V}=0 \equiv\{x \in$ $\left.\Omega \mid b^{T}(x-e) \geq 0\right\}$. We will denote this invariant set $\mathcal{Z}_{\Omega}$.

We can see that the solutions converge to the intersection of $\mathcal{Z}_{\Omega}$ with the set $\left\{x \in \Omega \mid b^{T}(x-e)=0\right\}$. Indeed, the solutions of (4) can only converge to a set of nonwandering points [5]. Considering the continuously differentiable function

$$
Z(x)=-b^{T} A^{-1} \ln (x)
$$

whose time-derivative is

$$
\dot{Z}=-b^{T} A^{-1} A(x-e)-b^{T} A^{-1} b u=-b^{T}(x-e) \leq 0
$$


when $x \in \mathcal{Z}_{\Omega}$, LaSalle's invariance principle indicates that

$$
\lim _{t \rightarrow+\infty} b^{T}(x(t)-e)=0
$$

for all solutions with initial condition in $\mathcal{Z}_{\Omega}$ (whose invariance indicates that $x(t)$ stays in $\left.\mathcal{Z}_{\Omega}\right)$. Therefore, any $x \in \mathcal{Z}_{\Omega}$ such that $b^{T}(x-e)>0$ cannot be a nonwandering point, so that we deduce that all solutions of (4) with $u$ given in (5) converge to a subset $\mathcal{M}_{\Omega}$ of $\left\{x \in \Omega \mid b^{T}(x-e)=0\right\}$.

This set $\mathcal{M}_{\Omega}$ contains the equilibrium $x=e$. It must now be examined if other solutions preserve the invariance of the hyperplane $b^{T}(x-e)=0$ because those invariant solutions would be candidates to attract the interconnected system (4)-(5). We will then analyze the system on the hyperplane with $u=0$, which is the value of the feedback when $b^{T}(x-e)=0$.

We are able to handle two cases:

$\mathbf{n}=\mathbf{2}$ In this case, the global asymptotic stability of $e$ is trivial: if a solution, other than $x(t)=e$ stays on the hyperplane, it is at the intersection of the hyperplane and a level set of the Lyapunov function $V(x)(>V(e))$. In this case, this intersection results in two disconnected points of the hyperplane. Invariance of the hyperplane on these points cannot be achieved because they are no equilibria. The only invariant solution of the hyperplane is then the equilibrium $e$, which is therefore globally asymptotically stable.

$\mathbf{n}=4$ We here only consider the case where $b=\alpha \mathbf{1}$ (for other forms of $b$, our work is still in progress). The time derivative of $W(x)=\mathbf{1}^{T}(x-e)$ (we drop the $\alpha$ factor) is

$$
\dot{W}=x^{T}(A(x-e)+\alpha \mathbf{1} u)=e^{T} A x+\alpha \mathbf{1}^{T} x u
$$

Along an invariant solution inside the hyperplane, this time derivative must be 0 , so that we must have $e^{T} A x=0$. Differentiating $e^{T} A x$ inside the hyperplane results in

$$
e^{T} \operatorname{Adiag}(x) A(x-e)
$$

If it exists, an invariant solution inside the hyperplane must then satisfy

$$
\begin{array}{ll}
\mathbf{1}^{T}(x-e)=0 & (a) \\
e^{T} A x=0 & (b) \\
e^{T} \operatorname{Adiag}(x) A(x-e)=0 & (c)
\end{array}
$$

We will now characterize the set defined by those three equalities.

The intersection of the hyperplane (a) and (b) define a two-dimensional plane in the 4-dimensional space. Indeed, they intersect in $e$ (so that we know that they intersect in the positive orthant) and they are not identical because (a) does not contain the origin while (b) contains the origin. We can then isolate two of the four coordinates $\left(x_{a}, x_{b}\right)$ (not necessarily $\left(x_{1}, x_{2}\right)$ ) and isolate them in $(a)-(b)$ as functions of the other two coordinates $\left(x_{c}, x_{d}\right)$ :

$$
\left(\begin{array}{l}
x_{a} \\
x_{b}
\end{array}\right)=C_{1}\left(\begin{array}{l}
x_{c} \\
x_{d}
\end{array}\right)+C_{2} e
$$


where $C_{1} \in \mathbb{R}^{2 \times 2}$ and $C_{1} \in \mathbb{R}^{2 \times 4}$ We then replace $\left(x_{a}, x_{b}\right)$ with this function in $(c)$, so that $(c)$ is now a two-dimensional quadratic form. The set of all the potentially invariant solutions inside the hyperplane $\mathbf{1}^{T}(x-e)$ is then a quadratic form, that is an ellipse, a hyperbola or a parabola (containing $e$ ). Let us now consider a solution $x(t)$ that would belong to this ellipse, this parabola, or the branch of the hyperbola that contains $e$. Because those are one dimensional objects containing a single equilibrium, any solution converges to the equilibrium in forward time or in backward time: we have

$$
\lim _{t \rightarrow+\infty} x(t)=e \quad \text { or } \quad \lim _{t \rightarrow-\infty} x(t)=e
$$

This results in

$$
\lim _{t \rightarrow+\infty} V(x(t))=V(e) \quad \text { or } \quad \lim _{t \rightarrow-\infty} V(x(t))=V(e)
$$

This is not possible, unless $x(t)=e$ because $V(x(t))$ must stay constant on the hyperplane ( $u=0$ implies $\dot{V}=0$ ). This unacceptable behavior is illustrated on the branch $H_{1}$ of the hyperbola that is shown on Figure 1: the potential solution crosses the level sets of $V$, which is unacceptable.

Similarly, if $x(t)$ is a solution that stays on the branch of the hyperbola that does not contain $e$, it will slide indefinitely on that branch and go to infinity (staying in the positive orthant or intersecting the borders of the orthant). This results in $V(x(t))$ going to infinity, which is impossible because $V$ must stay constant. This unacceptable behavior is illustrated on the branch $\mathrm{H}_{2}$ of the hyperbola that is shown on Figure 1: the potential solution goes to the border $x_{d}=0$, so that $V$ goes to infinity, which is unacceptable.

Those solutions sliding along those objects cannot exist, so that they do not belong to the largest invariant set in $\mathbf{1}^{T}(x-e)$, which we have then shown to be reduced to the equilibrium $e$. This equilibrium is therefore globally asymptotically stable.

For now, our analysis is limited to the case $n=2$ and $n=4$ (with $b=\alpha \mathbf{1}$ ) because, in more general cases, the computation of the time-derivatives of $W$ results in a quadratic form for $\dot{W}$, a cubic form for $\ddot{W}, \ldots$ whose intersection is hard to compute, and is the object of current research.

The approach that is developed in this paper is a Jurdjevic-Quinn approach to the control problem. However, in the proof of Proposition 2, we take a practical approach to show that the equilibrium is globally asymptotically stable by going back to the LaSalle principle, instead of showing the stability by computing the Lie Algebra that is used by Jurdjevic-Quinn [6].

\section{Application}

We will now consider two simple case of Lotka-Volterra systems in order to illustrate the efficiency of our controller. 


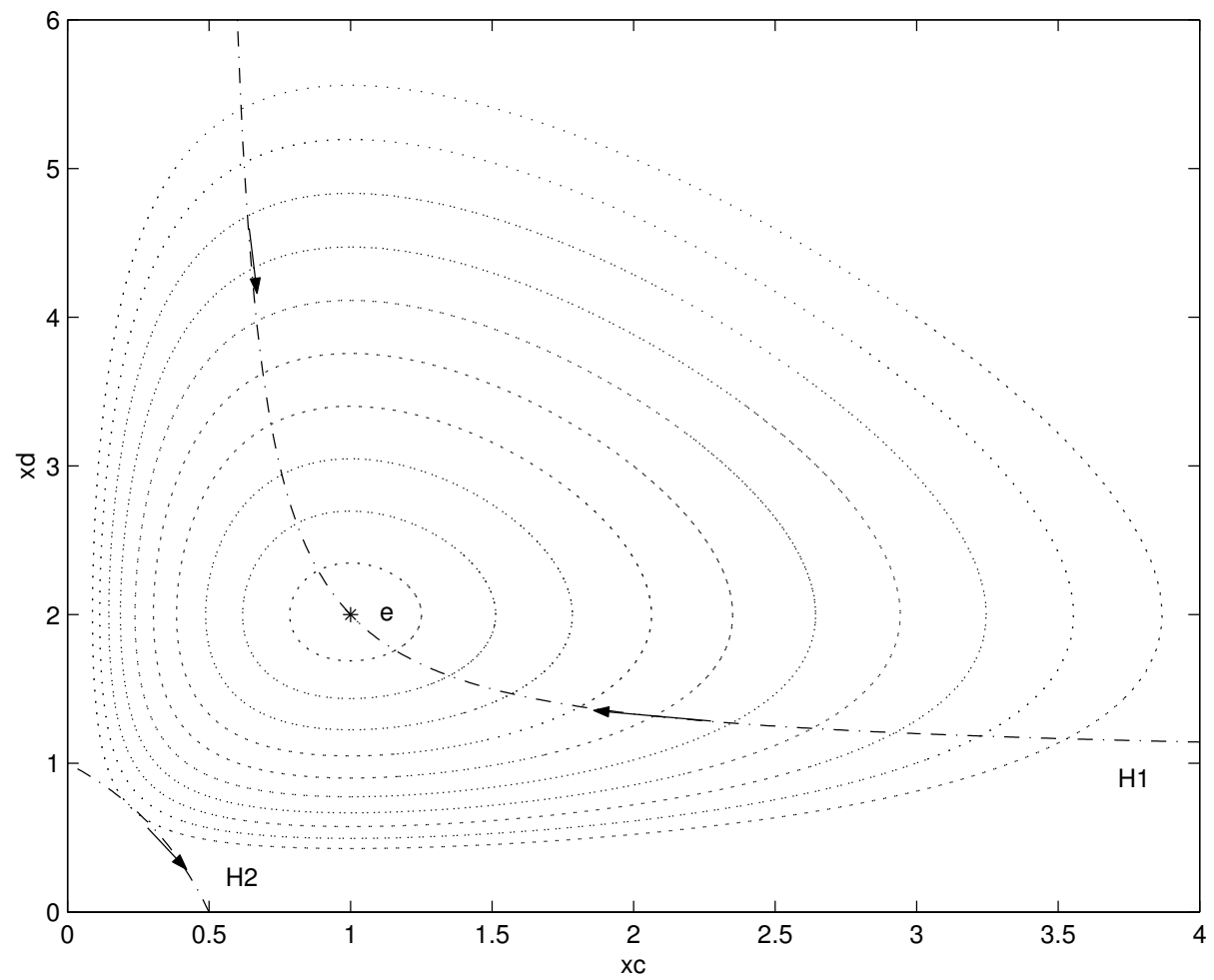

Figure 1: Level sets of $V(x)$ (dotted-lines) and branches of hyperbola (dash-dotted lines) that intersect, showing that no invariant solution other than $x(t)=e$ is possible inside the hyperplane $\mathbf{1}^{T}(x-e)=0$ 
The first one is a "one predator-one prey" system in the form

$$
\begin{aligned}
& \dot{x}_{1}=x_{1}\left(x_{2}-2-2 u\right) \\
& \dot{x}_{2}=x_{2}\left(-x_{1}+1-u\right)
\end{aligned}
$$

where $x_{1}$ is the predator and $x_{2}$ is the prey. A single control action is available, which acts on the death-rate of the predator (increase it with $u \geq 0$ ) and the growth-rate of the prey (decrease it with $u \geq 0$ ). For example, this could be a pesticide that both kills predators and preys.

As suggested in (5), the control action that will be applied is

$$
u=\sigma\left(2\left(x_{1}-1\right)+\left(x_{2}-2\right)\right)
$$

where we here define

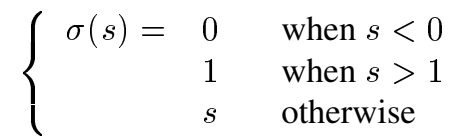

For an initial condition in $x_{0}=(3,4)$, this results in the behavior illustrated on Figures 2 and 3. Figure 2 shows that, when the solution is above the separating line $2\left(x_{1}-\right.$ $1)+\left(x_{2}-2\right)$ a control action is taken and the solution crosses the level set of $V(x)$, but when the solution is below this line, no control action is taken so that the solution follows a level set of $V(x)$. This is further illustrated by Figure 3: the time-evolution of $x_{1}$ and $x_{2}$ show that convergence actually takes place, while the evolution of $u$ and $V$ show that, between time 0.5 and time 3 , no control action is taken (because it would result in an increase of $V$ ), so that $V$ stays constant during that time-span; the rest of the time, $V$ decreases along the solution.

We will now illustrate our controller on a more intricate system containing two super-predator $\left(x_{1}\right.$ and $\left.x_{2}\right)$, two preys $\left(x_{3}\right.$ and $\left.x_{6}\right)$ and two intermediates of the foodchain $\left(x_{4}\right.$ and $\left.x_{5}\right)$, for which we have

$$
\begin{aligned}
& \dot{x}_{1}=x_{1}( \\
& \dot{x}_{2}=x_{2}( \\
& \dot{x}_{3}=x_{3}\left(-4 x_{1}\right. \\
& \dot{x}_{4}=x_{4}\left(\begin{array}{ll}
-3 x_{1} & -2 x_{2}
\end{array}\right. \\
& \dot{x}_{5}=x_{5}\left(-3 x_{2}\right. \\
& \dot{x}_{6}=x_{6}( \\
& \begin{array}{ccccccc}
+4 x_{3} & +3 x_{4} & & & -13 & -u & ) \\
& +2 x_{4} & +3 x_{5} & & -9 & -u & ) \\
& & & & +4 & & ) \\
& & +x_{5} & +4 x_{6} & -10 & & ) \\
& -x_{4} & & +5 x_{6} & -11 & & ) \\
& -4 x_{4} & -5 x_{5} & & +17 & & )
\end{array}
\end{aligned}
$$

We also see, on that model, that we can only act on the system by controlling the superpredator population. The target equilibrium is $e=(1,2,1,3,1,4)^{T}$. As suggested in this paper, we then apply a controller in the form

$$
u=\sigma\left(x_{1}+x_{2}-3\right)
$$

which results in the stabilization of the system. The behavior of the system is illustrated in Figure 4. We see that the two species that are illustrated $\left(x_{1}\right.$ and $\left.x_{6}\right)$ converge to their equilibrium value (the other 4 species also converge). We can also see that the solution is quite oscillatory, with $u$ alternating between 0 and positive values during 


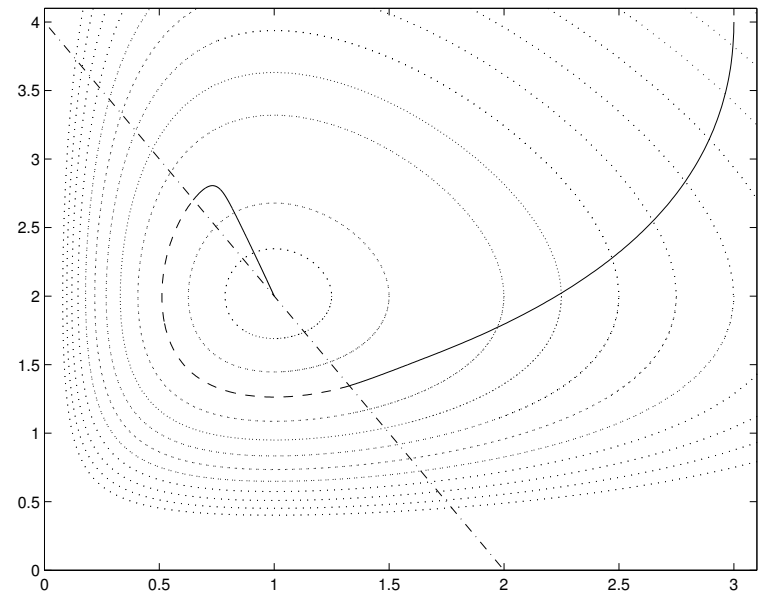

Figure 2: Phase-plane of the controlled Lotka-Volterra system (6). The dash-dotted line represent the separating "hyperplane" $b^{T}(x-e)=0$, the dotted lines represent the level sets of $V(x)$ and the remaining line represents a solution of $(6)$ with $x_{0}=(3,4)$ as initial condition (it is solid when a control is applied and dashed when $u=0$ )
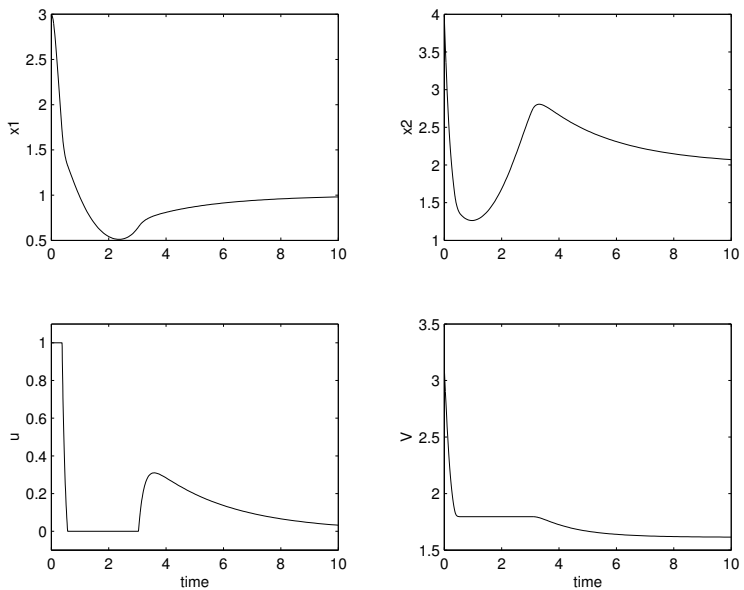

Figure 3: Time-evolution of $x_{1}, x_{2}, u$ and $V$ for the controlled system (6) with $x_{0}=$ $(3,4)$ as initial condition 
the first 15 time-units and then oscillating at positive values before converging to zero. Corresponding to that observation, we see that $V$ decreases in steps, its value staying constant every time $u$ equals 0 .

This system illustrates the fact (that was already evidenced in two-dimensions in [2]) that it is possible to regulate the pest (the preys) by controlling the predator. Also, as long as the equilibrium value and the model are well-known, the form of (8) also illustrates that we only need to know the value of the species that are acted upon $\left(x_{1}\right.$ and $x_{2}$ in this case).

Eliminating the necessity of knowing the exact value of the equilibrium in order to compute the constant term in (8) is the object of further research. We will try and introduce some adaptation in the control law so that the control law can be written

$$
u=\sigma\left(x_{1}+x_{2}-\eta(t)\right)
$$

with $\eta(t)$ converging to 3 , and the stability of the equilibrium is preserved.
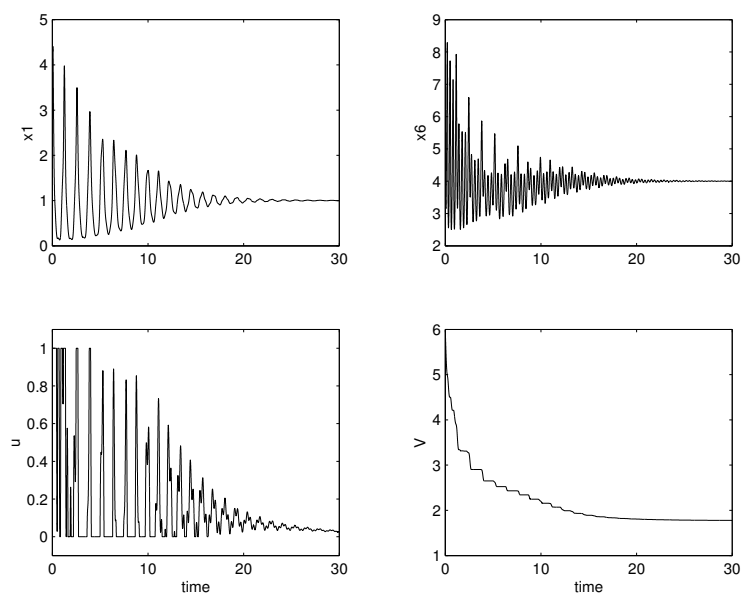

Figure 4: Time-evolution of $x_{1}, x_{6}, u$ and $V$ for the controlled system (7) with $x_{0}=$ $(3,4,2,5,3,6)$ as initial condition

\section{Conclusion}

In this paper, we have introduced a control law for the stabilization of the LotkaVolterra predator-prey system through positive feedback. The stability analysis is based on LaSalle's invariance principle and is limited to a few cases. The extension of the analysis to the case where $n$ is large (which applications in Section 4 show to be working) and the introduction of adaptation in the control law is the object of further research. 


\section{References}

[1] P. DeLeenheer and D. Aeyels. Accessibilty properties of controlled Lotka-Volterra systems. In Proceedings of the 39nth IEEE Conf. on Decision and Control, pages 3977-3981. Sydney, USA, 2000.

[2] B.S. Goh, G. Leitmann, and T.L. Vincent. Optimal control of a prey-predator system. Mathematical Biosciences, 19:263-286, 1974.

[3] J.L. Gouzé. Global behavior of $n$-dimensional Lotka-Volterra systems. Mathematical Biosciences, 113:231-242, 1993.

[4] J.L. Gouzé. Global stabilization of n-dimensional population models by a positive control. In Proceedings of the 33rd IEEE Conf. on Decision and Control, pages 1335-1336. Orlando, USA, 1994.

[5] J. Guckenheimer and P. Holmes. Nonlinear oscillations, dynamical systems, and bifurcations of vector fields. Springer-Verlag, New-York, 1983.

[6] V. Jurdjevic and J.P. Quinn. Controllability and stability. J. Differential Equations, 28:381-389, 1978.

[7] V.B. Kolmanovskii and N.I. Koroleva. Control of a predator-prey system with intraspecies competition. J. Appl. Math. Mech, 55:588-595, 1991.

[8] V. Volterra. Variations and fluctuations in the number of coexisting animal species (1927). In F.M. Scudo and J.R. Ziegler, editors, The golden age of theoretical ecology: 1923-1940, pages 65-234. Springer-Verlag, Berlin, 1978. 